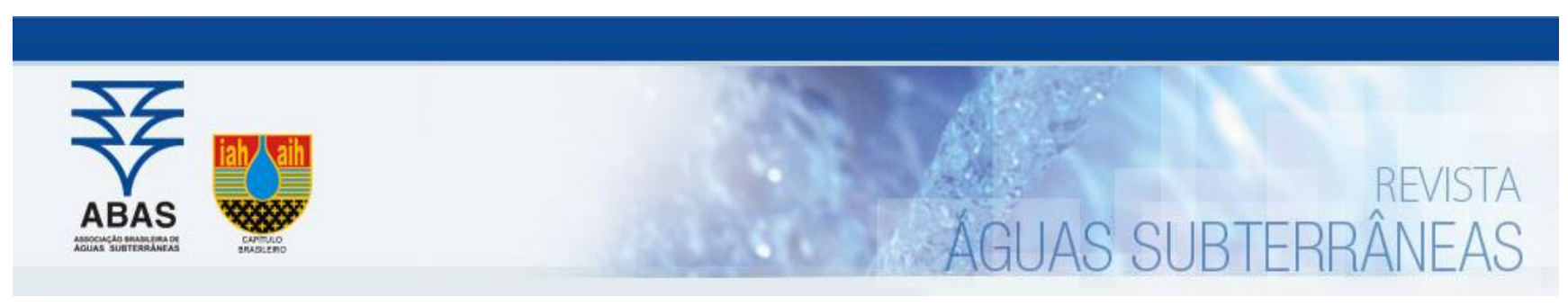

Artigos

\title{
Modelagem da vulnerabilidade e do risco de contaminação de águas subterrâneas em locais de disposição de resíduos sólidos urbanos
}

\section{Vulnerability and risk modeling of groundwater contamination in areas used for urban solid waste disposal}

\author{
Tiago de Morais Faria Novais; Mauro César Cardoso Cruz²; Mônica de Abreu Azevedo ${ }^{\bowtie}$ \\ 1 Universidade do Estado de Minas Gerais (UEMG), Divinópolis, MG; Centro Universitário de Formiga, Formiga, MG \\ 2 Universidade do Estado de Minas Gerais (UEMG), Divinópolis, MG \\ 3 Universidade Federal de Viçosa (UFV), Viçosa, MG
}

凶tiago.novais@uemg.br, mauro.cruz@uemg.br, monica.azevedo@ufv.br

\section{Palavras-chave: \\ Contaminação de solos. Áreas degradadas. \\ Resíduos sólidos urbanos.}

Keywords:

Soil contamination.

Degraded areas.

Urban solid waste.

Revisado por pares.

Recebido em: 25/03/2019.

Aprovado em: 25/05/2019.

\begin{abstract}
Resumo
Tendo em vista a necessidade de propiciar estudos que validem metodologias de análise de impactos ambientais, pretendeu-se, neste estudo, relacionar o potencial poluidor de um vazadouro a céu aberto, situado em um município brasileiro, com a vulnerabilidade da área a qual está inserido, utilizando um fator de potencial poluidor (I_p, como fator de risco de contaminação) e o modelo DRASTIC de análise de susceptibilidade de águas subterrâneas. O local escolhido como estudo de caso para este trabalho foi a área utilizada para a disposição final dos resíduos sólidos urbanos produzidos no município de Divinópolis, oeste do Estado de Minas Gerais, Brasil. O local de disposição pode ser caracterizado como um vazadouro a céu aberto, por não possuir nenhum mecanismo de controle das diversas formas de poluição ocasionadas. Ao aplicar a espacialização do índice DRASTIC na área do estudo, obtiveram-se os valores 76 a 79 , o qual demonstra que, apesar da variação nos valores, atribui-se valores do índice DRASTIC que caracterizam a área como de baixa vulnerabilidade à contaminação de águas subterrâneas. 0 índice de poluição obtido foi de 15,1, demonstrando uma elevada capacidade de contaminação, remetendo a áreas cuja intervenção imediata se mostra necessária.
\end{abstract}

Abstract

Considering the necessity to provide studies that validate methodologies for the analysis of environmental impacts, the purpose of this study was to relate the polluting potential of an open dump, located in a Brazilian municipality, with the vulnerability of the area which it is inserted, using a potential pollutant factor (I_p, as a risk factor for contamination) and the DRASTIC model of groundwater susceptibility analysis. The site chosen as a case study for this analysis was an area used for disposal of municipal solid waste produced in the municipality of Divinópolis, west of Minas Gerais state, Brazil. The disposal site can be characterized as an open dump because it has no control mechanism for the various forms of pollution caused. When applying the DRASTIC index to the study area, values between 76 and 79 were obtained, which show that, despite the variation in values, the DRASTIC index values characterize the area as being of low vulnerability to contamination groundwater. The obtained pollution index was 15.1, showing a high capacity of contamination, referring to areas whose immediate intervention proves necessary.

DOI: http:/dx.doi.org/10.14295/ras.v33i3.29526

\section{INTRODUÇÃO}

No Brasil, conforme relatado pela ABRELPE (2014), o número de áreas caracterizadas como lixões reduziu de 2.620, em 2000, para 1.569 em 2014. Conforme a PNSB de 2008 (IBGE, 2010), cerca de 50,8\% dos municípios brasileiros dispõem seus resíduos em lixões (2.862 municípios), 22,5\% em aterros controlados (1.252 municípios) e 27,7\% em aterros sanitários (1.541 municípios). Este panorama preocupante caracteriza o grande potencial poluidor que esta atividade implica ao meio ambiente e que deve se tornar alvo não só de políticas públicas, mas também de pesquisas acadêmicas norteadas à sua solução.

A capacidade poluidora dos chamados vazadouros a céu aberto (lixões) potencializa-se em virtude do entorno no qual está inserido. A poluição do ar, da água e do solo é proporcional ao tipo e ao volume de material, bem como à permeabili- 
dade do material da base do local de disposição final, à existência do sistema de drenagem de líquidos percolados (oriundos da degradação bioquímica dos resíduos) e de drenagem de gases, a mecanismos de controle de vetores de doenças e a outras medidas atenuadoras do potencial de degradação ambiental destes dispositivos urbanos (DAVOLI et al., 2010; PALMIOTTO et al., 2014). O atendimento a essas condições citadas depende das legislações e normatizações da região na qual o local de disposição está inserido. No Brasil, a norma NBR 13.896 (ABNT, 1997), que define os parâmetros para a implantação de aterros sanitários, dispõe condições relativamente generalizadas para a mitigação dos impactos ambientais. Condições essas que não definem o tipo de material de recobrimento, de material drenante, da técnica de tratamento do líquido percolado e, principalmente, não exige, de forma clara, a metodologia e periodicidade do monitoramento das condições hidrogeológicas do aterro sanitário.

As técnicas e dispositivos de construção e operação de um aterro sanitário (tido como o método de disposição adequada mais utilizado) existem com a função de mitigar os impactos ambientais relacionados à natureza do material depositado que, no caso, por possuir uma vasta gama de substâncias e compostos químicos e biológicos (componentes dos resíduos sólidos urbanos), apresentam um fator de risco ao meio ambiente e à saúde humana. Atribui-se o conceito de risco ao conceito de probabilidade de um ou mais poluentes advindos do local de disposição de RSU virem a alterar a qualidade de um recurso natural ou impactar a saúde humana de populações vizinhas (CONAMA, 2009; BUTT, 2011, SARAIVA et al., 2018).

A avaliação do risco oferecido pelo vazadouro ao seu entorno depende, de certa forma, do conceito de impacto ambiental atribuído em sua análise. Ao se considerar a degradação da qualidade das águas subterrâneas, Li et al. (2012), Galitskaya et al. (2014) e Gwenzi (2016), consideram que fatores topográficos, geológicos, geotécnicos e climáticos são predominantes na análise do risco atribuído ao local de disposição, porém são dependentes das condições de operação do aterro ou vazadouro (neste caso, inexistência de operação adequada) e, consequentemente, variáveis ao longo de um determinado tempo. Assim, Demirbilek et al. (2013) e Butt (2014) reiteram que o potencial poluidor de um determinado aterro de RSU, no que tange o aspecto qualidade de águas subterrâneas, é uma variável a ser quantificada em diferentes recortes temporais e passíveis de atenuação ou agravamento, seja de forma natural ou em virtude de ação humana.

Outros parâmetros de impacto ao meio ambiente podem ser a emissão de gases nocivos à atmosfera, conforme estudado por Ravindra et al. (2015), que analisou a dispersibilidade de materiais particulados e odor oriundos de um aterro em Chandigarh, na Índia. Um estudo realizado por Liu (2014) avaliou a estabilidade de taludes, enquanto a mobilidade de metais pesados dentro do corpo do aterro foi estudada por Aydi (2015). Contudo, o impacto na saúde humana e a percepção da população da magnitude desses impactos têm sido objeto de discussão e quantificação por parte de trabalhos como os realizados por Liu et al. (2016) e Mishra et al. (2017), que analisaram os impactos à saúde de populações vizinhas à áreas utilizadas para a disposição final de resíduos sólidos urbanos, considerando como cenários ambientais: o lixão ou vazadouro a céu aberto, as unidades de triagem e compostagem e o aterro sanitário. Liu et al. (2016) realizaram esta análise de riscos à saúde humana em populações vizinhas à um aterro sanitário que, mesmo caracterizado como uma forma admissível de disposição de resíduos, permanece como um empreendimento ambientalmente impactante.

A quantificação do risco de um determinado impacto ambiental associado ao local de disposição de RSU está condicionada à utilização de modelos matemáticos, fluxogramas, questionários ou outras ferramentas que auxiliem na compreensão das alterações do espaço físico no qual o vazadouro ou aterro está inserido. No entanto, cada metodologia de quantificação de risco limita-se a determinados parâmetros de contaminação (água subterrânea, superficial, partículas inaláveis, dentre outras) e a informações disponíveis para a sua avaliação. Assim, tem-se como área de estudo, a análise da vulnerabilidade de determinada área geográfica e o potencial poluidor de um local de disposição de resíduos.

A comparação entre a vulnerabilidade de contaminação de águas subterrâneas e o fator de impacto poluidor de um aterro de resíduos sólidos, tem sido, nas últimas décadas, discutida e objeto de metodologias estipuladas por pesquisadores e/ou agências ambientais governamentais. Neste aspecto, o modelo DRASTIC (ALLER et al., 1987) se destaca como um modelo de análise da vulnerabilidade de determinada área, caracterizado como um sistema paramétrico que utiliza de variáveis hidrogeológicas que influenciam na susceptibilidade da área a infiltrações e, consequentemente, nos danos ambientais ocasionados pela contaminação por fluidos. Esse método tem obtido aceitação no meio acadêmico em diferentes locais de estudo, sendo aplicado por Al-Adamat et al. (2003), Babiker et al. (2005) e Zhang (2016) em regiões com variadas configurações litológicas, geológicas, pedológicas e climáticas.

Quanto à avaliação do impacto poluidor de determinado empreendimento, vários são os Índices de Fator de Poluição $\left(I_{p}\right)$, caracterizados pelo risco de contaminação de águas subterrâneas (RAPTI-CAPUTO et al., 2006; YU, 2008; ZHANG et al., 2016). Esses índices diferem entre si conforme a natureza do empreendimento, sendo a disposição de RSU um importante meio de contaminação de recursos hídricos.

Tendo em vista a necessidade de propiciar estudos que validem metodologias de análise supracitadas, pretende-se neste trabalho, relacionar o potencial poluidor de um vazadouro a céu aberto, situado no município de Divinópolis, Oeste do estado de Minas Gerais (Brasil), com a vulnerabilidade da área a qual está inserido, utilizando um fator de potencial poluidor ( $I_{p}$, como fator de risco de contaminação) e o modelo DRASTIC (como índice de vulnerabilidade), comparando seus resultados a áreas avaliadas nos estudos de autores que utilizaram, previamente, essas metodologias.

\section{MATERIAL E MÉTODOS}

\section{1. Índice de Vulnerabilidade DRASTIC}

O modelo de análise de vulnerabilidade de áreas conhecido como DRASTIC (acrônimo que remete aos parâmetros analisa 
dos) foi proposto pela primeira vez, em 1987, pela Agência de Proteção Ambiental norte americana, a USEPA (United States Environmental Protection Agency) (ALLER et al., 1987). Este modelo tem sido comumente utilizado em estudos de áreas onde há disponibilidade de informações geográficas e hidrogeológicas, como os trabalhos realizados por Al-Adamat et al. (2003), Babiker et al. (2005) e Zhang et al. (2016), mostrando-se, portanto, uma metodologia aplicável a diferentes regiões do planeta. Assim, nesse método, são considerados como parâmetros indicadores de vulnerabilidade, as seguintes variáveis: profundidade da água subterrânea na região, a capacidade de infiltração do solo, a geologia do local, o tipo de solo, a topografia, o impacto na zona vadosa e a condutividade hidráulica do solo. A Equação 1 correlaciona o índice DRASTIC com as variáveis e seus respectivos pesos de consideração.

$$
\begin{aligned}
& \text { DRASTIC }=D * W_{D}+R * W_{R}+A * W_{A}+S * W_{S}+T * \\
& W_{T}+I * W_{I}+C * W_{C}
\end{aligned}
$$

$D, W_{D}$ Classe da profundidade da água subterrânea e seu peso, respectivamente

$R, W_{R}$ Classe da capacidade de infiltração e seu peso, respectivamente

$A, W_{A}$ Classe da litologia do local e seu peso, respectivamente

$S, W_{S}$ Classe do tipo de solo do local e seu peso, respectivamente

$T, W_{T}$ Classe da topografia da superfície do local e seu peso, respectivamente

$I, W_{I}$ Classe do impacto na zona vadosa e seu peso, respectivamente

$C, W_{C}$ Classe da condutividade hidráulica do solo do local e seu peso, respectivamente

Cada um dos sete parâmetros analisados recebe uma classificação numérica que varia de 1 a 10, conforme as condições hidrogeológicas do local, e cada fator é multiplicado por um peso, que varia de 1 a 5 , conforme a importância de cada quesito (ZHANG, 2016). A Tabela 1 ilustra a variação destas classificações e pesos, conforme denotado por Aller et al. (1987). Após as análises das condicionantes requeridas pelo método, o índice DRASTIC deve ser calculado e comparado em três di- ferentes categorias: índices menores que 135, denotam uma baixa vulnerabilidade da área; índices entre 135 e 150, representam média vulnerabilidade e, índices maiores que 150, indicam uma alta vulnerabilidade do local a impactos ambientais relativos às águas subterrâneas.

\section{2. Índice de Poluição (Ip)}

O Índice de Poluição caracteriza-se como uma análise probabilística paramétrica que atribui a fatores de interesse, valores numéricos correspondentes a sua interferência no sistema. Assim, parâmetros que influenciam a contaminação do solo e, consequentemente, das águas subterrâneas, são hierarquizados e padronizados segundo o peso de influência no sistema. Dessa forma, obtém-se um valor adimensional cujo objetivo é comparar o risco que diferentes áreas de disposição final de RSU oferecem ao seu entorno. No presente estudo, avalia-se o risco relacionado à contaminação do solo pela percolação (de) líquidos oriundos de precipitação atmosférica e degradação biológica e química dos resíduos aterrados, com base nos parâmetros, valores e pesos propostos por Rapti-Caputo (2006). A Equação 2 descreve o cálculo do referido índice.

$$
I_{p}=\sum_{i=1}^{8} W_{i} * R_{i}
$$

Em que W refere-se ao peso do parâmetro de risco (importância do parâmetro, variando de 1 a 5) e $\mathrm{R}$ o fator de risco quanto aos quesitos (a) volume de resíduos aterrados, (b) o destino dos líquidos percolados, (c) tipo de resíduo, (d) estado físico do resíduo, (e) a biodegradabilidade dos resíduos, (f) a forma de monitoramento do local, (g) a compactação dos resíduos e (h) o material de recobrimento do aterro/vazadouro. A Tabela 2 evidencia os valores para $\mathrm{R}$ e o peso $\mathrm{W}$ para cada um dos oito parâmetros analisados neste estudo.

Conforme Rapiti-Caputo et al. (2006), aterros/vazadouros com índice Ip menor que 3 apresentam um baixo potencial poluidor, podendo estar localizados em qualquer área; índice Ip entre 3 a 6 podem estar situados em áreas de média vulnerabilidade; índices entre 6 e 9 podem estar situados em áreas de baixa vulnerabilidade e aqueles que apresentam índices maiores que 10 devem ter uma intervenção imediata quanto à mitigação de seu impacto às águas subterrâneas. 
Tabela 1 - Valores e pesos dos parâmetros do Índice DRASTIC.

\begin{tabular}{|c|c|c|c|}
\hline Fator & Intervalo/Características & Valor & Peso \\
\hline \multirow{7}{*}{$\begin{array}{c}\text { D } \\
\text { Profundidade da água } \\
\text { subterrânea(m) }\end{array}$} & $0-1,5$ & 10 & \multirow{7}{*}{5} \\
\hline & $1,5-4,6$ & 9 & \\
\hline & $4,6-9,3$ & 7 & \\
\hline & $9,3-15$ & 5 & \\
\hline & $15-23$ & 3 & \\
\hline & $23-30$ & 2 & \\
\hline & $>30$ & 1 & \\
\hline \multirow{5}{*}{$\begin{array}{c}\mathrm{R} \\
\text { Recarga (mm/dia) }\end{array}$} & $0-50$ & 1 & \multirow{5}{*}{4} \\
\hline & $50-100$ & 3 & \\
\hline & $100-175$ & 6 & \\
\hline & $175-250$ & 8 & \\
\hline & $>250$ & 9 & \\
\hline \multirow{9}{*}{$\begin{array}{c}\text { A } \\
\text { Litologia }\end{array}$} & Folhelho Maciço & $1-3$ & \multirow{9}{*}{3} \\
\hline & Rochas ígneas e metamórficas & $2-5$ & \\
\hline & Rochas ígneas e metamórficas alteradas & $3-5$ & \\
\hline & Sucessões finamente acamadas de arenitos, calcários e & $5-9$ & \\
\hline & Arenitos maciços & $4-9$ & \\
\hline & Calcários maciços & $4-9$ & \\
\hline & Areias e cascalhos & $6-9$ & \\
\hline & Basaltos & $2-10$ & \\
\hline & Calcários e Cársticos & $9-10$ & \\
\hline \multirow{9}{*}{$\begin{array}{c}\text { S } \\
\text { Tipo de Solo }\end{array}$} & Argila não expansiva & 1 & \multirow{9}{*}{1} \\
\hline & Franco argiloso & 3 & \\
\hline & Silto arenoso & 4 & \\
\hline & Franco & 5 & \\
\hline & Franco arenoso & 6 & \\
\hline & Argila expansiva & 7 & \\
\hline & Solo arenoso & 9 & \\
\hline & Solo com cascalho & 10 & \\
\hline & Solo fino & 10 & \\
\hline \multirow{5}{*}{$\begin{array}{c}\mathrm{T} \\
\text { Declividade do terreno } \\
(\%)\end{array}$} & $0-2$ & 10 & \multirow{5}{*}{1} \\
\hline & $2-6$ & 9 & \\
\hline & $6-12$ & 5 & \\
\hline & $12-18$ & 3 & \\
\hline & $>18$ & 1 & \\
\hline \multirow{10}{*}{ Zona Vadosa } & Argilas e siltes & $1-2$ & \multirow{10}{*}{5} \\
\hline & Xistos & $2-5$ & \\
\hline & Calcários & $2-7$ & \\
\hline & Arenitos & $4-8$ & \\
\hline & Calcários, arenitos e folhelhos & $4-8$ & \\
\hline & Areias e cascalhos com teor significativo de silte e argila & $4-8$ & \\
\hline & Rochas ígneas e metamórficas & $2-8$ & \\
\hline & Areias e cascalhos & $6-9$ & \\
\hline & Basaltos & $2-10$ & \\
\hline & Calcários cársticos & $8-10$ & \\
\hline \multirow{6}{*}{$\begin{array}{c}\text { C } \\
\text { Condutividade hidráulica } \\
\text { (m/dia) }\end{array}$} & $1-4,1$ & 1 & \multirow{6}{*}{4} \\
\hline & $4,1-12,2$ & 2 & \\
\hline & $12,2-28,5$ & 4 & \\
\hline & $28,5-40,7$ & 6 & \\
\hline & $40,7-81,5$ & 8 & \\
\hline & $>81,5$ & 10 & \\
\hline
\end{tabular}

Fonte: Aller et al. (1987); Zhang et al. (2016) 
Tabela 2 - Fatores de determinação do Índice de Poluição Ip

\begin{tabular}{|c|c|c|c|}
\hline Risco & Peso (W) & Classe & $\mathbf{R}$ \\
\hline \multirow{4}{*}{ Volume de resíduos disposto } & \multirow[t]{4}{*}{, } & $<10$ toneladas/dia & 0,1 \\
\hline & & 10 a 50 toneladas/dia & 0,2 \\
\hline & & 50 a 500 toneladas/dia & 0,4 \\
\hline & & > 500 toneladas/dia & 1 \\
\hline \multirow{4}{*}{$\begin{array}{l}\text { Sistema de drenagem de per- } \\
\text { colado }\end{array}$} & \multirow{4}{*}{5} & Drenagem externa e interna existente & 0,1 \\
\hline & & Drenagem interna existente & 0,3 \\
\hline & & Reuso do percolado no próprio sistema & 0,5 \\
\hline & & Drenagem ausente & 1 \\
\hline \multirow{4}{*}{ Tipo de resíduos } & \multirow{4}{*}{3} & Inerte* & 0,1 \\
\hline & & Urbano & 0,5 \\
\hline & & Industrial - não perigoso & 0,8 \\
\hline & & Perigoso & 1 \\
\hline \multirow{4}{*}{ Estado físico dos resíduos } & \multirow{4}{*}{3} & Solidificado com a matriz inerte & 0,1 \\
\hline & & Sólido & 0,2 \\
\hline & & Lodo com umidade menor que $70 \%$ & 0,5 \\
\hline & & Lodo com umidade maior que $70 \%$ & 1 \\
\hline \multirow{4}{*}{ Tipologia de estabilização } & \multirow{4}{*}{2} & Não biodegradável & 0,1 \\
\hline & & Aeróbico & 0,3 \\
\hline & & Aeróbico e anaeróbico & 0,5 \\
\hline & & Anaeróbico & 1 \\
\hline \multirow{4}{*}{ Sistema de monitoramento } & \multirow{4}{*}{2} & Poço de monitoramento e geomembrana & 0,1 \\
\hline & & Geomembrana & 0,3 \\
\hline & & Poço de monitoramento & 0,5 \\
\hline & & Ausente & 1 \\
\hline \multirow{4}{*}{ Compactação dos resíduos } & \multirow{4}{*}{1} & Compactado com equipamento pneumático & 0,1 \\
\hline & & Compactado com trator de esteira & 0,2 \\
\hline & & Compactado manualmente & 0,5 \\
\hline & & Sem compactação & 1 \\
\hline \multirow{4}{*}{ Recobrimento final } & \multirow{4}{*}{1} & Solo compactado com argila & 0,1 \\
\hline & & Argila compactada & 0,2 \\
\hline & & Solo não compactado & 0,5 \\
\hline & & Ausente & 1 \\
\hline
\end{tabular}

Fonte: Rapti-Caputo et al. (2006)

* Resíduos inertes e urbanos diferenciados na metodologia: resíduos urbanos contemplam os potencialmente recicláveis mais matéria orgânica

\section{3. Área de estudo}

O local escolhido como estudo de caso foi a área utilizada para a disposição final dos resíduos sólidos urbanos produzidos no município de Divinópolis, oeste de Minas Gerais, situado nas coordenadas $20^{\circ} 08^{\prime} 20^{\prime \prime} \mathrm{S}$ e $44^{\circ} 53^{\prime} 02^{\prime \prime}$ W. O local de disposição pode ser caracterizado como um vazadouro a céu aberto, por não possuir nenhum mecanismos de controle das diversas formas de poluição ocasionadas. 0 referido vazadouro opera desde o início da década de 1980, conforme informações fornecidas pelo poder público municipal. Na Figura 1 é ilustrada a localização do vazadouro e as curvas de nível da topografia da área, obtidas na Secretaria Municipal de Planejamento Urbano e Meio Ambiente (SEPLAM).

A coleta de informações que possibilitou a análise da vulnerabilidade da área e do fator de impacto às águas subterrâneas, como proposto nesta pesquisa, baseou-se em mapas temáticos e trabalhos prévios de órgãos e agências governamentais dedicadas ao estudo e avaliação hidrológica, geológica, meteorológica e afins. Assim, com a utilização do mapa hidrogeológico do Brasil (CPRM, 2014), do mapa hidrogeológico do Estado de Minas Gerais (CETEC, 1981), do atlas digital das águas de Minas (UFV, 2011), do banco de solos de Minas Gerais (FEAM, 2013), foi possivel obter os parâmetros necessários à aplicação da metodologia DRASTIC. Além das variáveis supracitadas, as informações relativas a dois poços tubulares do tipo cisterna, localizados próximos à área de estudo, foram obtidas no Sistema de Informação e Gestão de Águas Subterrâneas, SIAGAS (CPRM, 2016), especificados na Tabela 3, os quais possibilitaram a determinação da profundidade do nível e da capacidade de recarga de águas subterrâneas, conforme discriminado na Equação 3. 
Figura 1 - Localização e topografia da área de estudo

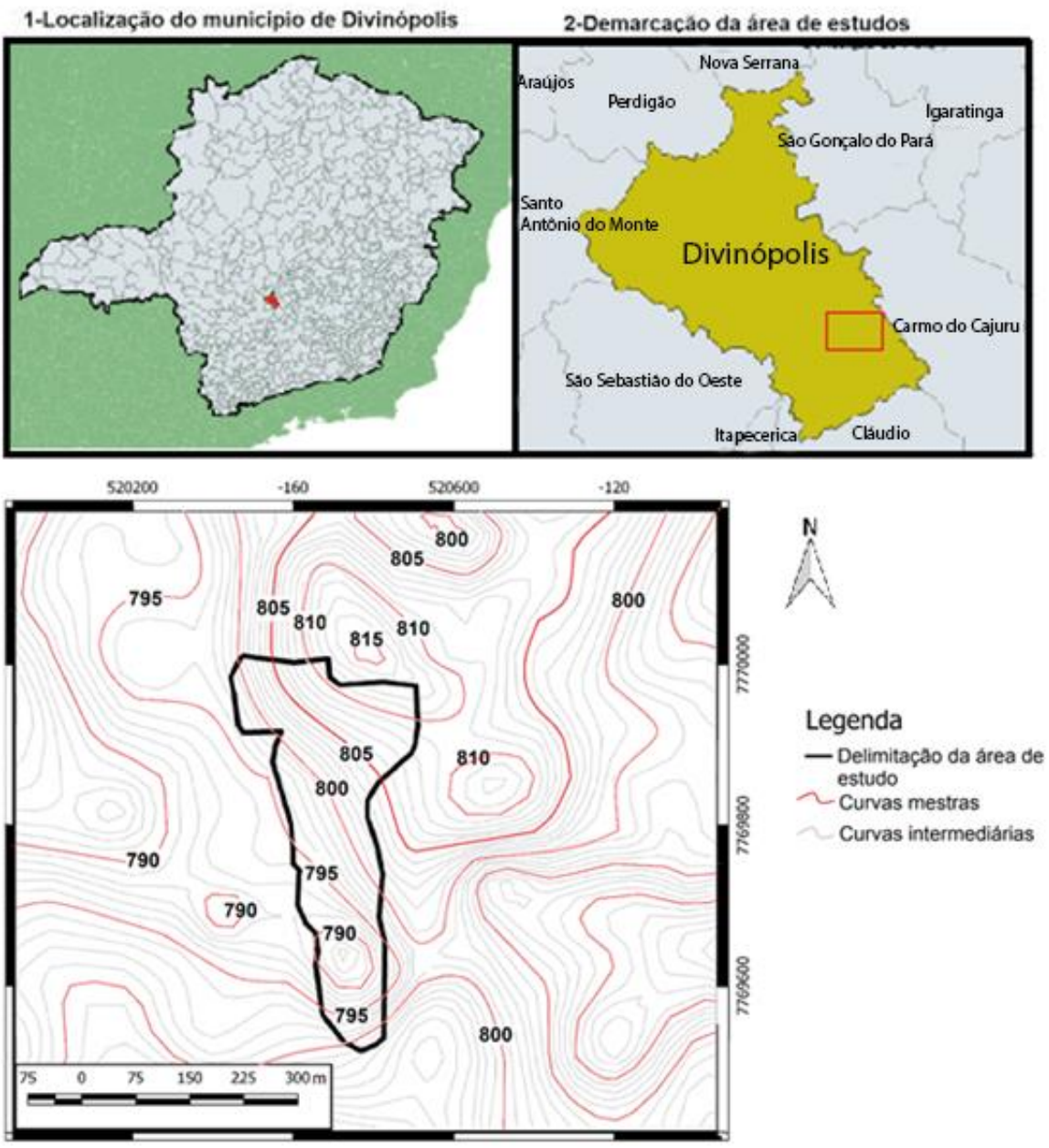

Tabela 3 - Poços tubulares próximos ao local de estudo cadastrados no SIAGAS

\begin{tabular}{cccc}
\hline \multirow{2}{*}{ Nome } & \multirow{2}{*}{ Número do ponto } & \multicolumn{2}{c}{ Coordenadas (metros)* } \\
& & Longitude (E) & Latitude (N) \\
\hline Ferrador & 3100021350 & 520896,35 & 7767972,82 \\
Chácara Beira & 3100021351 & 509895,92 & 7770036,14 \\
Rio & & & \\
\hline
\end{tabular}

* Sistema de coordenadas WGS 84 - UTM Fuso 23

$$
R=S_{y} \cdot \Delta H / \Delta t
$$

Em que Sy representa o rendimento específico do meio poroso saturado e $\Delta \mathrm{H}$ e $\Delta \mathrm{t}$, a variação da altura do nível de água em um determinado tempo, respectivamente.

Para a obtenção das variáveis acerca do local de disposição de resíduos sólidos (volume disposto diariamente, operação, monitoramento e compactação dos resíduos, além do sistema de drenagem), informações advindas do poder público municipal, por intermédio da SEPLAM, além de levantamentos topográficos e fotográficos da empresa Viasolo S/A (responsável pelo gerenciamento de resíduos e disposição no município) que proporcionaram a determinação dos parâmetros necessários para formulação do índice poluidor $I_{p}$.

\section{RESULTADOS E DISCUSSÃO}

Avaliando as informações obtidas nos mapas hidrogeológicos do Brasil e de Minas Gerais (CETEC, 1981; CPRM, 2014) é possível concluir que a região em que se situa o vazadouro de RSU (assim como toda a área do município de Divinópolis) está compreendida em um embasamento fraturado indiferenciado, composta predominantemente por rochas metamórficas. Essa informação, corroborada pelo trabalho de Carneiro e Barbosa (2008), permitiu a caracterização do parâmetro "A" do índice de vulnerabilidade, atribuindo-se o valor 5 a esta variável.

A tipologia do solo da região pode ser considerada, segundo o mapa de solos do estado de Minas Gerais (FEAM, 2013), como argissolo vermelho-amarelo distrófico na área do vazadouro, encontrado como solo arenoso em camadas superficiais e argiloso com o aumento da profundidade analisada. Assim, atribuiu-se o valor de 6 para a zona vadosa, valor definido para solos argilo-siltosos compreendida como o tipo de solo susceptível à percolação abaixo dos resíduos, definido no parâmetro "I". No entanto, o solo superficial caracteriza-se como um solo areno-siltoso, proveniente de jazidas próximas ao aterro, de coloração clara, de elevada permeabilidade e au- 
sente de compactação. Essas informações permitem a estimativa do parâmetro "S", definido como a classificação do solo superficial, que influencia na capacidade de percolação de água no solo, o qual neste estudo foi atribuído o valor 4 (Tabela 1).

As considerações acerca da profundidade do nível de água subterrânea puderam ser realizadas apenas com as informações obtidas no SIAGAS, conforme descrito nos materiais e métodos, e foram extraídas de dois poços de captação de água, os quais apresentaram uma profundidade média de 67 $\mathrm{m}$ até o nível estático de água. Essa profundidade considerável atenua o fator de vulnerabilidade em larga escala para o índice proposto, o qual, neste parâmetro ("D") pôde ser atribuído o valor 1.

Utilizando-se da classificação do solo da zona vadosa, relacionado como um solo argissolo vermelho-amarelo foi possível estimar condutividade hidráulica do referido meio com a avaliação de estudos prévios de outros pesquisadores deste tema (Tabela 4).

Tabela 4 - Valores de condutividade hidráulica para solos argissolos vermelho-amarelo

\begin{tabular}{ccc}
\hline Autor & Rocha & K (cm/s) \\
\hline Oliveira et al. (2010) & Gnaisse & $4,44 \times 10^{-5}$ \\
Pereira et al. (2013) & Gnaisse & $9,19 \times 10^{-6}$ \\
Vieira et al. (2015) & Gnaisse & $1,00 \times 10^{-6}$ \\
\hline
\end{tabular}

Apesar das diferenças de condutividade hidráulica apresentadas nos estudos citados, todos os valores apresentam condutividade hidráulica menor que $1 \mathrm{~m} / \mathrm{d}$, definindo-se como variável de valor igual a 1 (parâmetro $\mathrm{C}$ ). Com esse valor de condutividade hidráulica e com o tipo de solo da zona vadosa, pôde ser definida a capacidade de recarga das águas subterrâneas, discriminada na Equação 3, sendo obtido um valor de 150 mm/dia (parâmetro "R").

Na Tabela 5 apresenta-se um resumo das informações anteriormente descritas e necessárias para a aplicação do modelo DRASTIC, juntamente com os fatores relacionados a cada parâmetro obtido.

Ao aplicar a espacialização do índice DRASTIC obtido na Tabela 5 (uma vez que há uma variação nos valores) na área do estudo, obteve-se o mapa da Figura 2, o qual demonstra que, apesar da variação nos valores, atribui-se valores do índice DRASTIC que caracterizam a área como de baixa vulnerabilidade à contaminação de águas subterrâneas.

Conforme descrito previamente, nos materiais e métodos, os valores 76 a 79 para o índice DRASTIC denotam uma área com baixa vulnerabilidade de contaminação de águas subterrâneas, também obtido por Yu et al. (2010) em uma área de semelhantes características. Este valor para o referido índice pode ser justificado, entre outros valores, pela baixa permeabilidade do solo e pela profundidade do nível do lençol freático, os quais obtiveram valores atribuídos relativamente baixos, além do peso dos referidos parâmetros ser de grande magnitude na determinação do índice.

Quanto ao vazadouro, segundo levantamento topográfico realizado pela Viasolo S/A (Viasolo Engenharia Ambiental S/A, Betim, Minas Gerais), este possui declividade média entre 8 e $10 \%$. O referido local de disposição recebe, diariamente, cerca de 165 toneladas de resíduos sólidos urbanos, não sendo recebidos resíduos provenientes de estabelecimentos de saúde e da construção civil. Neste vazadouro inexiste qualquer tipo de drenagem interna de percolados e de águas pluviais externas às valas de operação. 0 recobrimento final das valas de disposição ocorre semanalmente utilizando-se de solo obtido na superfície do próprio local, caracterizado por uma granulometria arenosa e sem compactação. Ressalta-se que durante a operação, não são compactados os resíduos dispostos no local, e que também inexiste qualquer forma de monitoramento do vazadouro. A Tabela 6 resume as informações relativas ao vazadouro, cujos valores do índice de poluição estão atribuídos para cada parâmetro, bem como dos pesos de consideração.

Tabela 5 - Resumo dos valores considerados no Índice DRASTIC

\begin{tabular}{clccc}
\multicolumn{1}{c}{ Característica da área } & Valor atribuído & Peso (W) & Fator*W \\
\hline Fator & Profundidade do nível de água subterrânea - 67 & 1 & 5 & 5 \\
D & m (> 30m) & 6 & 4 & 24 \\
$\mathrm{R}$ & Capacidade de infiltração 100 a 175 mm & 5 & 3 & 15 \\
$\mathrm{~S}$ & Rochas metamórficas & 4 & 5 & 20 \\
$\mathrm{~T}$ & Solo superficial silto arenoso & 5 a 1 & 1 & 5 a 1 \\
I & Declividade média entre 10 e 30\% & 6 & 1 & 6 \\
$\mathrm{C}$ & Zona vadosa com predominância de argila & 1 & 4 & 4 \\
\hline
\end{tabular}


Figura 2 - Mapa de vulnerabilidade da área segundo Índice DRASTIC.

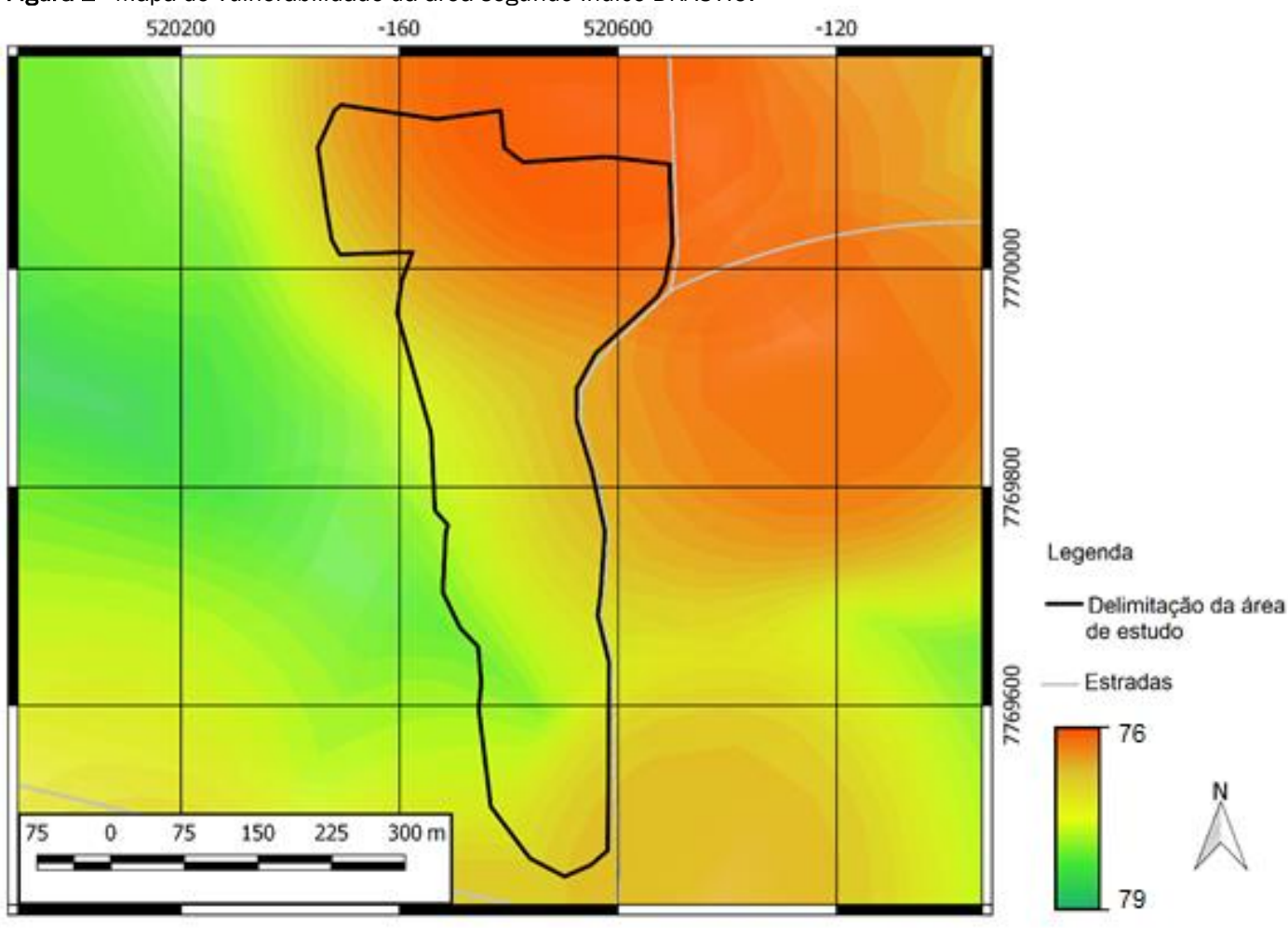

Tabela 6 - Resumo dos valores considerados no Índice de Poluição Ip

\begin{tabular}{lcccc}
\hline \multicolumn{1}{c}{ Fator de risco } & Situação & $\mathbf{R}$ & $\mathbf{W}$ & $\mathrm{R} * \mathbf{W}$ \\
\hline Volume de resíduos aterrados & 160 ton/d & 0,4 & 5 & 2 \\
Sistema de drenagem & Inexistente & 1 & 5 & 5 \\
Tipo de resíduo & Urbano & 0,5 & 3 & 1,5 \\
Estado físico dos resíduos & Sólido & 0,2 & 3 & 0,6 \\
Biodegradablidade dos resíduos & Anaeróbica & 1 & 2 & 2 \\
Monitoramento do local & Inexistente & 1 & 2 & 2 \\
Compactação dos resíduos & Sem compactação & 1 & 1 & 1 \\
Material de recobrimento final & Solo sem compactação & 1 & 1 & 1 \\
\hline
\end{tabular}

O índice de poluição demonstra uma elevada capacidade de contaminação e, segundo Rapiti-Caputo (2006), valores acima de 10 remetem à áreas cuja intervenção imediata se mostra necessária, uma vez que a exposição dos recursos naturais da área às substâncias nocivas, oriundas do processo de decomposição dos resíduos, possui elevados riscos qualitativos e quantitativos. O valor de 15,1 para o Ip justifica-se pelos parâmetros relativos à ausência do sistema de drenagem, à biodegradabilidade dos resíduos, à ausência de monitoramento do local, à ausência de compactação dos resíduos e ao material de recobrimento final apresentarem a situação menos favorável dentro das possibilidades apresentadas na metodologia, caracterizando um local de disposição com condições de operação invariavelmente inadequadas.

Pode-se ressaltar que, apesar do índice de susceptibilidade DRASTIC se caracterizar, neste caso, como relativamente baixo, em decorrência do profundo nível de água e baixa permeabilidade do solo, o potencial poluidor encontrado para o empreendimento localizado nesta área evidencia a importân- cia da análise de ambas as vertentes desta fase do gerenciamento de resíduos sólidos: a escolha da área e operação do local de disposição final, sem os quais não se pode afirmar mitigação dos impactos ocasionados por aterros de resíduos sólidos.

\section{CONCLUSÕES}

A avaliação da susceptibilidade de uma área passível de recebimento de resíduos sólidos caracteriza uma etapa importante na sua escolha durante a etapa de implantação de um aterro sanitário. No entanto, esta etapa comumente é ignorada ou reduzida à verificação do atendimento a critérios de simplicidade demasiada, como no caso das exigências da NBR 13.896 (ABNT 1997), em comparação aos critérios analisados pela metodologia DRASTIC. Na referida metodologia, analisada nesta pesquisa, as variáveis de observação são consideradas de forma empírica, mas que não necessitam de complexos ensaios de caracterização ou quantificação, sendo de relativo fácil acesso por banco de dados governamentais, 
ampliando sua aplicação, mas usualmente não existe na escala adequada.

Quanto à análise do potencial poluidor pelo índice de poluição Ip, caracterizado nesta pesquisa, evidencia a importância da correta operação dos sistemas de disposição final de resíduos sólidos, como o recobrimento periódico, a coleta de gases, drenagem de percolados, compactação de resíduos, entre outros. A importância destes critérios de operação na metodologia de quantificação do potencial poluidor, apresentada neste trabalho, reforça a necessidade de fiscalizações mais criteriosas destes locais, uma vez que sua inadequada condução pode acarretar em danos irreversíveis ao meio ambiente. Embora a susceptibilidade da área tenha sido considerada baixa, mostra-se necessária uma intervenção imediata no local.

Estas duas análises, vulnerabilidade e potencial poluidor, reforçam a necessidade da revisão dos processos de licenciamento de áreas de disposição final de resíduos sólidos, uma vez que os atuais critérios e parâmetros analisados são de reduzida profundidade, realçada por recentes metodologias de análise destes empreendimentos, como os descritos nesta pesquisa.

\section{REFERÊNCIAS}

AL-ADAMAT, R. A. N., FOSTER, I. D. L., BABAN, S. M. J. Groundwater vulnerability and risk mapping for the Basaltic aquifer of the Azraq basin of Jordan using GIS, Remote sensing and DRASTIC. Applied Geography, v. 23, n. 4, p. 303-324, out. 2003.

ALLER, L., BENNETT, T., LEHR, J. H., PETTY, R. J., HACKETT, G. Drastic: a standardized system for evaluating ground water pollution potential using hydrogeologic settings. Dublin, Ohio: USEPA, 1987.

ASSOCIAÇÃO BRASILEIRA DE EMPRESAS DE LIMPEZA PÚBLICA E RESÍDUOS ESPECIAIS. Panorama dos Resíduos Sólidos no Brasil 2014. São Paulo: ABRELPE, 2014.

ASSOCIAÇÃO BRASILEIRA DE NORMAS TÉCNICAS. NBR 10.004: Resíduos Sólidos - Classificação. Rio de Janeiro: 2004. $71 \mathrm{p}$.

ASSOCIAÇÃO BRASILEIRA DE NORMAS TÉCNICAS. NBR 13.896: Aterros de resíduos não perigosos - Critérios para projeto, implantação e operação. Rio de Janeiro: 1997. 12 p.

AYDI, A. Assessment of heavy metal contamination risk in soils of landfill of Bizerte (Tunisia) with a focus on application of pollution indicators. Environmental Earth Sciences, v. 74, n. 4, p. 3019-3027, mar. 2015.

BABIKER, I. S., MOHAMED, M. A. A., HIYAMA, T., KATO, K. A GISbased DRASTIC model for assessing aquifer vulnerability in Kakamigahara Heights, Gifu Prefecture, central Japan. Science of The Total Environment, v. 345, n. 1-3 p. 127-140, jun. 2005.

BUTT, T. E., GOUDA, H. M., BALOCH, M. I., PAUL, P., JAVADI, A. A., ALAM, A. Literature review of baseline study for risk analysis
- The landfill leachate case. Environmental International, v. 63, p. 149-162, fev. 2014.

BUTT, T. E., INGLES, A. J. D., BALOCH, M. I. A Conceptual Model Outline for Integrated Exposure Assessment of Waste Disposal Sites. Environmental Progress \& Sustainable Energy, v. 30, n. 4, p. $696-708$, jan. 2011.

CARNEIRO, M. A., BARBOSA, M. S. C. Implicações Geológicas e Tectônicas da Interpretação Magnetométrica da Região de Oliveira, Minas Gerais. Revista Brasileira de Geofísica, v. 26, n. 1, p. 87-98, mar. 2008.

CETEC - FUNDAÇÃO CENTRO TECNOLÓGICO DE MINAS GERAIS. Mapa Hidrogeológico de Minas Gerais. Secretaria de Ciência e Tecnologia - Instituto de Geociências Aplicadas. Belo Horizonte, 1981. Escala 1:1.000.000.

CHANG, N. B., DAVILA, E. Municipal solid waste characterizations and management strategies for the Lower Rio Grande Valley, Texas. Waste Mangement, v. 28, n. 5, p. 776-794, jun. 2008.

CONSELHO NACIONAL DO MEIO AMBIENTE. Resolução No 420 de dezembro de 2009. Dispõe sobre critérios e valores orientadores de qualidade do solo quanto à presença de substâncias químicas e estabelece diretrizes para o gerenciamento ambiental de áreas contaminadas por essas substâncias em decorrência de atividades antrópicas.

CPRM - SERVIÇO GEOLÓGICO DO BRASIL. Mapa Hidrogeológico do Brasil. Ministério de Minas e Energia - Secretaria de Geologia, Mineração e Transformação Mineral. Brasília, 2014. Escala 1:5.000.000.

CPRM - SERVIÇO GEOLÓGICO DO BRASIL. Sistema de Informações de Águas Subterrâneas. Brasília, 2016. Disponível em: http://siagasweb.cprm.gov.br/layout/. Acesso em: 20 jan. 2019.

CURRIE, J., GREENSTONE, M., MORETTI, E. Superfund Cleanups and Infant Health. American Economic Association: Papers and Proceedings, v. 101, n. 3, p. 435-441, mar. 2011.

DAVOLI, E., FATTORE, E., PAIANO, V., COLOMBO, A., PALMIOTO, M., ROSSI, A. N., GRANDE, M. II., FANELLI, R.. Waste management health risk assessment: A case study of a solid waste landfill in South Italy. Waste Management, v. 30, n. 8-9, p. 1608-1613, ago/set. 2010.

DEMIRBILEK, D., ÖNAL, A. Ö., DEMIR, V., USLU, G., ARSLANOGLU-ISIK, H., 2013. Characterization and pollution potential assessment of Tunceli, Turkey municipal solid waste open dumping site leachates. Environmental Monitoring and Assessment, v. 185, n. 11, p. 9435-9449, nov. 2013.

FUNDAÇÃO ESTADUAL DO MEIO AMBIENTE (Minas Gerais). Banco de Solos de Minas Gerais. Belo Horizonte, 2013. Disponivel em: http://www.feam.br/noticias/1/949-mapas-desolo-do-estado-de-minas-gerais. Acesso em 20 jan. 2019. 
GALITSKAYA, I. V., PUTILINA, V. S., YUGANOVA, T. I. Substantiation of Managerial Decisions for Minimizing the Risk of Groundwater Contamination at the Sites of Solid Municipal Waste Disposal. Water Resources, v. 41, n. 7, p. 844-853, nov. 2015.

GOUVEIA, N., PRADO, R. R. Riscos à saúde em áreas próximas a aterros de resíduos sólidos urbanos. Revista de saúde pública, v. 44, n. 5, p. 859-866, set. 2010.

GWENZI, W., GORA, D., CHAUKURA, N., TAURO, T. Potential for leaching of heavy metals in open-burning bottom ash and soil from a non-engineered solid waste landfill. Chemosphere, v. 147, p. 144-154, mar. 2016.

IBGE - Instituto Brasileiro de Geografia e Estatística. Pesquisa Nacional de Saneamento Básico - PNSB, 2010.

LAL, R. M., NAGPURE, A. S., LUO, L., TRIPATHI, S. N., RAMASWAMI, A., BERGIN, M. H., RUSSELL, A. G. Municipal solid waste and dung cake burning: discoloring the Taj Mahal and human health impacts in Agra. Environmental Research Letters, v. 11, n. 10, p. 1-8, out. 2016.

LEBERSORGER, S., BEIGL, P. Municipal solid waste generation in municipalities: Quantifying impacts of households structure, commercial waste and domestic fuel. Waste Management, v. 31, n. 9-10, p. 1907-1915, set/out. 2011.

LI, Y., LI, J., CHEN, S., DIAO, W. Establishing indices for groundwater contamination risk assessment in the vicinity of hazardous waste landfills in China. Environmental Pollution, v. 165, p. 77-90, mar. 2012.

LIU, Y., PENG, R., XU, Y., NAI, C., DONG, L., REN, J. Mechanical behavior of typical hazardous waste and its influence on landfill stability during operation. Journal of Material Cycles and Waste Management, v. 16, n. 4, p. 597-607, jun. 2014.

LIU, Y., LIU, Y., LI, Y., FU, X., GUO, H., MENG, R., LU, W., ZHAO, M., WANG, H. Health risk impacts analysis of fugitive aromatic compounds emissions from the working face of a municipal solid waste landfill in China. Environment International, v. 97, p. 15-27, dez. 2016.

MISHRA, H., KARMAKAR, S., KUMAR, R., JITENDRA, S. A Framework for Assessing Uncertainty Associated with Human Health Risks from MSW Landfill Leachate Contamination. Risk Analysis: An International Journal, v. 37, n. 7, p.1237-1256, jul. 2017.

OLIVEIRA, L. A., GONÇALVES, R. M., MARTINS, F. P. Contraste de condutividade hidráulica em solos de texturas arenosa e argilosa encontrados nos tributários da margem esquerda do rio tijuco, município de Ituiutaba, estado de Minas Gerais, Brasil. Caminhos de Geografia, v. 11, n. 33, p. 230-243, mar. 2010.
PALMIOTTO, M., FATTORE, E., PAIANO, V., CELESTE, G., COLOMBO, A., DAVOLI, E. Influence of a municipal solid waste landfill in the surrounding environment: Toxicological risk and odor nuisance effects. Environment International, v. 68, p. 1624, jul. 2014.

PEREIRA, A. C., PORTO FILHO, F. Q., FREITAS NETO, O., SEVERO, R. N. F. Avaliação da condutividade hidráulica para solos compactados utilizados em camadas selantes e análise de estabilidade de aterro para resíduos sólidos da perfuração de poços de petróleo. Holos, v. 4, p. 16-36, ago. 2013.

RAPTI-CAPUTO, D., SDAO, F., MASI, S. Pollution risk assessment based on hydrogeological data and management of solid waste landfills. Engineering Geology, v. 85, n. 1-2, p. 122131, mai. 2006.

RAVINDRA, K., KAUR, K., MOR, S. System analysis of municipal solid waste management in Chandigarh and minimization practices for cleaner emissions. Journal of Cleaner Production, v. 89 , p. $251-256$, fev. 2015.

SARAIVA, A. B., SOUZA, R. G., MAHLER, C. F., VALLE, R. A. B. Consequential lifecycle modelling of solid waste management systems - Reviewing choices and exploring their consequences. Journal of cleaner production, v. 202, p. 488-496, 2018.

UNIVERSIDADE FEDERAL DE VIÇOSA. Atlas Digital das Águas de Minas. Viçosa, 2011. Disponível em: http://www.atlasdasaguas.ufv.br/home.html. Acesso em 20 jun. 2016.

VIEIRA, B. C., FERREIRA, F. S., GOMES, M. C. V. Propriedades físicas e hidrológicas dos solos e os escorregamentos rasos na Serra do Mar Paulista. Ra'e Ga, v. 34, p. 269-287, ago. 2015.

YU C., YAO Y. Y., HAYES G., ZHANG B. X., ZHENG C. M. Quantitative assessment of groundwater vulnerability using index system and transport simulation, Huangshuihe catchment. Science of The Total Environment, v. 408, n. 24, p. 61086116, nov. 2010.

YU, L. An approach to the study on investigation, monitoring, prevention and control of groundwater Pollution in Beijing's plain area. Urban Geology, v. 3, p. 1-4, jun. 2008.

ZAMAN, A. U., LEHMANN, S. Urban growth and waste management optimization towards 'zero waste city'. City, Culture and Society, v. 2, n. 4, p. 177-187, dez. 2011.

ZHANG, B., LI, G., CHENG, P., YEH, T. C. J., HONG, M. Landfill Risk Assessment on Groundwater Based on Vulnerability and Pollution Index. Water Resources Management, v. 30, n. 4, p. 1465-1480, jan. 2016. 